\title{
INTERRELACIONES ENTRE GRAMATICALIZACIÓN Y FRASEOLOGÍA EN ESPAÑOL*
}

\author{
LEONOR RUIZ GURILLO \\ Universidad de Alicante. Grupo GRIALE
}

\section{INTRODUCCIÓN}

El cambio lingüístico es un hecho que siempre ha interesado a los lingüistas desde distintas perspectivas y corrientes. La Teoría de la Gramaticalización, que hunde sus raíces en aspectos cognitivos y discursivo-pragmáticos, nace a mediados de los años 80 con la intención de convertirse en un modelo de aplicación universal. Aunque no ha estado exenta de críticas, en los últimos años ha consolidado un conjunto de métodos que se han revelado útiles en las aplicaciones a lenguas concretas y que han guiado los análisis de los investigadores, de modo que se han proporcionado contraejemplos o se han perfilado nuevas líneas. La fraseología, por sus especiales características, constituye una porción de interés para la Gramaticalización. Si a ello se une que las propiedades de la fraseología y de la gramaticalización coinciden, se comprenderá el alcance que puede tener tal acercamiento.

En este artículo se investigan las interrelaciones entre gramaticalización y fraseología en español. Las herramientas teóricas de la primera proporcionan una explicación satisfactoria para las propiedades de fijación e idiomaticidad de la segunda. Las cadenas de gramaticalización, las diversas aproximaciones al cambio linguíístico, el basamento teórico para los marcadores del discurso, la propuesta de la subjetivación o la Teoría del Cambio Semántico a partir de Inferencias Asociadas son algunos de los aspectos que se ilustrarán a partir de la evolución de unidades fraseológicas concretas. Así, se ha elegido una locución marcadora (desde luego), una fórmula rutinaria (estaría bueno) y una locución verbal (cubrirse de gloria). Utilizamos ejemplos extraídos de los corpus CORDE y CREA (http://www.rae.es).

\footnotetext{
* Este artículo ha sido posible gracias al Proyecto de Investigación FFI 2008-00179/FILO «Aplicaciones a la clase de español como lengua extranjera de la ironía y el humor», financiado por el Ministerio de Educación y Ciencia y cofinanciado con fondos FEDER (2008-2011). Deseamos agradecer los diversos comentarios de Belén Alvarado Ortega que han servido para mejorar este trabajo.
} 


\section{FUNDAMENTOS DE LA TEORÍA DE LA GRAMATICALIZACIÓN}

Como decíamos, la gramaticalización es una teoría del cambio lingüístico que explica «the linguistic process, both through time and synchronically, of organization of categories and of coding» ${ }^{1}$. Esta definición, que no aporta nada nuevo a los estudios llevados a cabo sobre la evolución de las lenguas, entronca con diversos aspectos diferenciadores que cabe tener en cuenta. Por un lado, intenta explicar el porqué de los cambios linguiísticos, y para hacerlo propone unas cadenas de gramaticalización. De manera general, estas han sido recogidas por J. Bybee a partir de las elaboradas por T. Givón, B. Heine y M. Reh o Ch. Lehmann $^{2}$. En ellas se integran los diversos aspectos del cambio en los niveles morfológico (descategorización y reanálisis), fonológico (erosión), léxico, distribucional, de frecuencia y semántico (debilitamiento).

En líneas generales, estos principios de gramaticalización coinciden con las propiedades de la fraseología. Así, la fijación fraseológica se observa en el reanálisis (nivel morfológico), la erosión (nivel fonológico) o la fijación distribucional (nivel distribucional). Por su parte, la idiomaticidad fraseológica se refleja en el debilitamiento (nivel semántico). Vemos, pues, que las propiedades de la fraseología y los planteamientos teóricos que las sustentan podrían constituir un buen punto de partida para llevar a cabo estudios más profundos que refuercen los principios de gramaticalización.

Por otro lado, la aproximación global al fenómeno del cambio cuenta con diversos acercamientos que se pueden resumir a partir de la propuesta de $\mathrm{O}$. Fischer y A. Rosenbach ${ }^{3}$. El esquema tiene en cuenta tanto las aproximaciones sincrónicas como las diacrónicas, tanto los modelos funcionales como los tipológicos o los formales:

1 E. C. Traugott y B. Heine, «Introduction», en E. C. Traugott y B. Heine, eds., Approaches to grammaticalization, Amsterdam, John Benjamins, vol. I, 1991, pág. 1.

2 J. L. Bybee, «Mechanisms of semantic change in grammaticization», en L. De Stadler, ed., Third International Cognitive Linguistic Conference (Lovaina, 18-23 de julio de 1993), 1993; T. Givón, «From Discourse to Syntax: Grammar as a Processing Strategy», Syntax and Semantics, 12: Discourse and Syntax, 1979, págs. 81-112; B. Heine y M. Reh, Grammaticalization and reanalysis in African Languages, Hamburg, Helmut Buske, 1984; Ch. Lehmann, Thougths on grammaticalization, München, Lincom Europa, 1995.

3 O. Fischer y A. Rosenbach, «Introduction», en O. Fischer, A. Rosenbach y D. Stein, eds., Pathways of Change. Grammaticalization in English, Amsterdam, John Benjamins, 2000, pág. 14. 


\begin{tabular}{|c|c|c|c|}
\hline \multirow[t]{2}{*}{ Perspectiva } & \multicolumn{2}{|c|}{ Acercamientos a la gramaticalización } & \multirow[b]{2}{*}{ Formal } \\
\hline & Tipológico & Funcional & \\
\hline Sincrónica & $\begin{array}{l}\text { modelos } \\
\text { interlingüísticos }\end{array}$ & $\begin{array}{l}\text { base discursivo-pragmática } \\
\text { y cognitiva de la gramática }\end{array}$ & no se aplica \\
\hline $\begin{array}{l}\text { 'Cambio } \\
\text { en proceso' }\end{array}$ & & $\begin{array}{l}\text { variación sincrónica } \\
\text { criollos }\end{array}$ & $\begin{array}{l}\text { solo bajo una } \\
\text { hipótesis de } \\
\text { base doble }\end{array}$ \\
\hline Diacrónica & $\begin{array}{l}\text { evolución de } \\
\text { la gramática en } \\
\text { General }\end{array}$ & $\begin{array}{l}\text { evolución de las formas } \\
\text { ¿gramática emergente? }\end{array}$ & reanálisis \\
\hline
\end{tabular}

TABLA 1: Acercamientos a la gramaticalización, según O. Fischer y A. Rosenbach (pág. 14).

En este artículo adoptamos un acercamiento funcional. Proponemos ilustrarlo tanto desde el punto de vista sincrónico, esto es, teniendo en cuenta los aspectos discursivo-pragmáticos y cognitivos de la gramática, como desde el punto de vista diacrónico, es decir, haciendo referencia a la evolución de las formas en los diversos niveles de gramaticalización. No olvidamos el papel de las cadenas de gramaticalización y la idea de que el cambio es gradual y dinámico.

En el intento de conjugar aspectos de fraseología y de gramaticalización, partimos de una clasificación fraseológica comúnmente aceptada (colocaciones, locuciones y enunciados fraseológicos ${ }^{4}$ ) e ilustramos los grupos seleccionados con un análisis de la gramaticalización diferenciado. En el caso de las locuciones marcadoras, la cadena de gramaticalización propuesta por E. C. Traugott ${ }^{5}$ para los marcadores del discurso nos sirve de guía para desde luego. Por su parte, los enunciados fraseológicos, representados por estaría bueno, se explican a partir de la noción de subjetivación desarrollada por la autora a lo largo de diversos trabajos ${ }^{6}$. Finalmente, el análisis de las locuciones verbales se sirve de la Teoría del Cambio Semántico a partir de Inferencias Asociadas (Invited Inferencing Theory of Semantic Change, IITSC) ${ }^{7}$. El orden en la descripción de

4 G. Corpas, Manual de fraseología española, Madrid, Gredos, 1996.

5 E. C. Traugott, «On the rise of epistemic meanings in English: an example of subjectification in semantic change», Language, 65, 1989, págs. 31-55.

${ }^{6}$ E. C. Traugott, «From propositional to textual and expressive meanings: some semanticpragmatic aspects of grammaticalization», en W. P. Lehmann y Y. Malkiel, eds., Perspectives on Historical Linguistics, Amsterdam, John Benjamins, 1982, págs. 245-271; E. C. Traugott, «On the rise of epistemic meanings in English: an example of subjectification in semantic change», Language, 65, 1989, págs. 31-55; E. C. Traugott, From Etymology to Historical Pragmatics, SHEL, UCLA (Conferencia), 2000.

7 Esta propuesta ha sido desarrollada en trabajos como los de E. C. Traugott y R. B. Dasher, Regularity in Semantic Change, Cambridge, CUP, 2002, y L. Brinton y E. C. Traugott, Lexicalization and gramaticalization in language change, Cambridge, CUP, 2005. 
las unidades fraseológicas también supone un desarrollo cronológico de las teorías que van a sustentar tales análisis.

\section{LAS CADENAS DE GRAMATICALIZACIÓN Y LAS LOCUCIONES MARCADORAS}

Los marcadores del discurso han sido convenientemente integrados en la Teoría de la Gramaticalización a partir de la cadena propuesta por E. C. Traugott ${ }^{8}$ :

C. Circ. $>$ Adv. de frase $>$ Marcador del discurso

Figura 1. Evolución de los marcadores del discurso, según E. C. Traugott

De este modo, es previsible que, tras una fase intermedia, diversos complementos circunstanciales se gramaticalicen como marcadores del discurso de diversos tipos (estructuradores de la información, conectores, reformuladores, operadores o marcadores de control del contacto $^{9}$ ). Algunos de ellos son de carácter complejo, es decir, se definen como unidades fraseológicas. En concreto, se adscriben a lo que hemos denominado locución marcadora ${ }^{10}$, pues sirven para unir (sin embargo, en cambio, en consecuencia, de todas maneras), para ordenar (en primer lugar) o reformular (es decir, de hecho), para atenuar (de alguna manera) o intensificar (que no veas), o para mostrar acuerdo o desacuerdo (de acuerdo, por supuesto, desde luego, de ninguna manera, ni pensarlo, ni soñarlo). Ahora bien, para la Teoría de la Gramaticalización es posible establecer una última fase en aquellos marcadores del discurso que se especializan como matizadores o, más concretamente, como elementos que actúan en la relación entre hablante y oyente. Se habla entonces de un proceso de intersubjetivación. En el caso que nos ocupa, algunas locuciones marcadoras mostrarían usos como elementos de intersubjetivación entre hablante y oyente, lo que posibilitaría explicarlas como fórmulas rutinarias.

A continuación nos referimos a la cadena de gramaticalización y al proceso de fraseologización que ha sufrido el actual operador del discurso desde luego. Para J. Portolés ${ }^{11}$ es un operador de refuerzo argumentativo que presenta el miembro discursivo al que afecta como evidente. Por su parte, M. ${ }^{a}$ A. Martín Zorraquino y J. Portolés ${ }^{12}$ lo definen como un marcador de modalidad epistémica de «evidencia».

\footnotetext{
8 «On the rise of epistemic meanings in English», op. cit.

9 J. Portolés, Marcadores del discurso, Barcelona, Ariel, 1998.

${ }^{10}$ L. Ruiz Gurillo, Las locuciones en español actual, Madrid, Arco/Libros, 2001, págs. 56-58.

11 Op. cit., pág. 144.

${ }^{12}$ M. ${ }^{a}$ A. Martín Zorraquino y J. Portóles, «Los marcadores del discurso», en I. Bosque y V. Demonte, dirs., Gramática descriptiva de la lengua española, Madrid, Espasa, 1999, págs. 4051-4213.
} 
Nuestra tarea consiste básicamente en explicar cómo se ha pasado del circunstancial (libre) a la fórmula (fija) (fijación); y cómo los valores semánticos temporales han evolucionado hasta los epistémicos y evidenciales (idiomaticidad). Para ello, proponemos cuatro fases:

Fase I: desde luego es un circunstancial de carácter temporal que acompaña al verbo, puede situarse en posición postverbal o preverbal y se refiere a hechos temporales acaecidos con anterioridad. Así lo encontramos en (1), texto jurídico donde se dicta una pena por el incumplimiento de las ordenanzas:

(1)

Es nuestra voluntad \& asy lo declaramos que por ese mesmo fecho que qual quiera fiziere contra las dichas ordenanças / o contra qual quier dellas jncurra en la pena / o penas dellas. ypso jure. E desde luego se obligado a pagar la dicha pena / o penas dellas jnforo conçiençie syn que aya nin se espere otra condepnaçion quanto quier que el delicto sea oculto;

(CORDE. Anónimo, Ordenanzas reales de Castilla, 1484)

Nótese cómo se establece una relación contextual entre incumplir las ordenanzas y pagar la pena que se estipula: la relación es principalmente temporal, pues tras el incumplimiento se sucederá el pago de la pena ('desde entonces'); ahora bien, también es consecutiva, ya que a un hecho seguirá por ley el segundo ('como consecuencia'). Creemos que tal valor secundario es el que va a permitir la evolución del sintagma hasta la locución marcadora actual. Dos elementos cobran sentido desde este enfoque: por un lado, el núcleo del sintagma nominal, luego, es un adverbio de carácter temporal que, atendiendo a las propiedades de estratificación y divergencia ${ }^{13}$ también ha desarrollado funciones ilativas o consecutivas en español actual. Por otro lado, el enlace prepositivo desde expresa el punto de procedencia, por lo que establece un anclaje espacial, temporal o nocional.

Su carácter de circunstancial de tiempo le permite aparecer en posición preverbal con gran frecuencia, como ocurre en (1) y también (2): el vizcaíno va a ser engañado por Domingo, que le promete los favores de una cortesana si previamente le da a él un doblón de los dos que ha recibido de su tío. Por ello, Domingo considera desde ese momento que el doblón es suyo:

13 P. Hopper, «On some principles of grammaticalization», en E. C. Traugott y B. Heine, Approaches to grammaticalization, op. cit., págs. 17-35. 
(2)

El vizcaíno le agradeció mucho el placer que le hacía; y así le dijo que un doblón tenía que darla, de dos que su tío le había dado para sus necesidades. Crecióle el ojo á Domingo, y desde luego contó por suyo el dicho doblón, y aun los dos, y así, le previno que para prima noche le aguardase en su aposento, que él se la enviaría á él tan buena, y aun mejor que se la había prometido; pero que era necesario darla de antemano el doblón para tenerla grata. Así se lo prometió el vizcaíno. (CORDE. Alonso de Castillo Solórzano. Tardes entretenidas en seis novelas, 1625)

En los dos casos anteriores aparece precedido por la conjunción y. Con tales usos como circunstancial de tiempo se documenta bien entrado el siglo XIX, como ejemplifica $(3)^{14}$ :

\section{(3)}

Corría el año de 1260, cuando un venerable religioso conocido entonces por el nombre de Fr. Pedro de Valencia y venerado después con el de San Pedro Pascual fundó este convento, el primero de su orden que hubo en España. Perteneció su comunidad a la regla de Mercedarios calzados, y desde luego ocupó el mismo edificio del que aún se encuentran restos en el lugar donde en siglos anteriores se alzaban unas casas pertenecientes al ayuntamiento de la ciudad y una pequeña ermita. (CORDE. Gustavo Adolfo Bécquer, Historia de los templos de España, 1857)

Fase II: desde luego perfila sus valores ilativos o consecutivos. Aunque mantiene un significado temporal, permite reformular el enunciado en el que se inserta como la consecuencia de lo previo. Con tal uso aparece en (4), donde tiene el significado de 'como consecuencia, por lo tanto', ya que los nombramientos actuales revocan y anulan los previos:

\section{(4)}

(...) en caso de no hauer pariente de las dos lineas para las referidas puedan los testamentarios que se hallaren nombrar sacerdotes que las gocen, concurriendo con ellos su heredero el señor Don Manuel Vocangel su hermano a quien nombra por Patron vnico de dicho Patronato y memoria de missas, y despues de sus dias nombra por tal patron a sus hijos y descendientes en forma regular prefiriendo el mayor al menor y el baron a la hembra y en falta de los susodichos nombra a los hijos y descendientes de Don Geronimo Vocangel su hermano difunto en la misma forma, y en defecto de los susodichos al pariente suio mas cercano por linea materna, y que goce el que tuviere dicho patronato cinquenta ducados de vellon de renta; Y desde luego reuoca y annula los nombramientos de capellanes que hauia hecho para las referidas dos capellanias en su testamento cerrado, y nombra a sus parientes en la forma que va preuenido para que entre en el goce de dichas memorias

${ }^{14}$ Como nos informan M. ${ }^{\text {a }}$ A. Martín Zorraquino y J. Portolés, op. cit., pág. 4152, nota 107, Terreros y Pando documenta el uso de desde luego como temporal hasta el siglo XVIII. 
de missas y con el nombramiento de los testamentarios que vbiere y del $\mathrm{Pa}$ trono puedan tomar la possesion de ellas y perciuir sus frutos y rentas y cumplir con las cargas de su dotacion (CORDE. Anónimo, Codicilo de Eugenia Bocángel y Bolero [Documentos de la casa Bocangelina (1562-1710)], 1707)

El nombramiento de Don Manuel Vocángel como patrón del Patronato anula y revoca los anteriores, por lo que el enunciado encabezado por desde luego se convierte en la conclusión de todo el discurso previo. Ello permite la progresión del discurso, de modo que se pueden designar nuevos testamentarios. Sin embargo, los significados temporales siguen estando muy presentes en contextos como este ${ }^{15}$.

Fase III: desde luego actúa como un operador de refuerzo argumentativo. $\mathrm{Su}$ aparición en contextos consecutivos favorece la ilazón contextual entre lo previo y lo siguiente, de manera que permite reforzar el argumento en el que se inserta como la consecuencia más fuerte. Equivale a otros operadores de refuerzo como por supuesto ${ }^{16}$. Su posición es libre dentro del enunciado. En (5) el protagonista se ve en la obligación de reunir una junta provisional que pueda reestablecer el sistema de gobierno:

(5)

No intento causaros el menor trastorno, al paso que no dudo os prestareis con vuestras posibilidades en aquella parte que tendrá á bien detallaros la autoridad competente, á fin de que pueda yo mantener la fuerza armada. Voy desde luego á reunir una junta provisional del corregimiento, que merezca la confianza de los pueblos, con la cual espero tratar y establecer el sistema de gobierno que por de pronto sea más conforme para desterrar, si es posible, los abusos y arbitrariedades de que tanto se lamentan los buenos españoles (...) (CORDE. Antonio Pirala, Historia de la guerra civil y de los partidos liberal y carlista, I, 1868)

${ }^{15}$ El CORDE nos permite documentar una estructura con cierta fijación, como desde luego, que aparece entre 1539 y 1952. Abunda en documentos notariales y jurídicos y presenta un claro valor de reformulador parafrástico, como evidencia el siguiente ejemplo: «(...)hella la acetó y della husando anbos a dos juntos y de mancomún a boz de vno cada vno dellos de sus vienes de por sí e por el todo ynsolidum renunciaron las leyes de la mancomunidad de duobus res debendi y la auténtica presente hoc hita de fideyussoribus y el veneficio de la deuda y excursión y las demás deste caso como en ellas se contiene dijeron reciuían y reciuieron, acetaban y acetaron, y reciúan y reciuieron a la dicha Eugenia de Pontes por su hija abdotiba con todos los vienes profeticios y adbeticios y derechos que tienen y tubieren, de todo lo qual harán ynventario solemne y siempre la tratarán como si fuese su hija ligítima y natural, y la dejarán como desde luego la dejan e ynstituyen por su heredera vniversal en todos sus vienes rahíces e muebles, derechos y actiones que tienen y tubieren, y dellas le hicieron donación buena, pura, perfeta e yrrebocable quel derecho llama entre biuos y como mejor aproveche a la dicha Eugenia de Pontes (...)». (CORDE, Anónimo, Prohijamiento, 1619).

${ }_{16}$ Para un análisis detallado de por supuesto y de acuerdo, véase L. Ruiz Gurillo, «Por supuesto, estamos de acuerdo: un análisis argumentativo de los operadores de refuerzo», Oralia, 2, 1999, págs. 241-261. 
Así, el enunciado «voy desde luego á reunir una junta provisional de corregimiento» se presenta como un argumento reforzado por dicha locución marcadora, lo que facilita la progresión del discurso.

Fase IV: como operador de evidencia puede desarrollar valores de mediación entre hablante y oyente, es decir, de intersubjetivación: destacar la propia opinión frente a la del resto, mostrar acuerdo con el interlocutor, etc. Estos valores conviven en la sincronía actual con los propios de refuerzo argumentativo de la evidencia.

Resulta abundante la combinación de la unidad fraseológica con yo, lo cual manifiesta distancia de la propia opinión frente a otras. Esta función de contraste, propia de desde luego ${ }^{17}$ se manifiesta en ejemplos como el de (6), donde el protagonista, a pesar de su incertidumbre y sus vacilaciones, no duda en aceptar el duelo que se le propone:

(6)

Se acercaba el momento de que la Bella Vizcaína tenía que partir. Yo fui a la fragata a dirigir la maniobra y a ponerla en franquía, fuera de todos los barcos de la bahía de Cádiz. De allí volví en el bote. Me encontraba en la mayor incertidumbre.

Un acontecimiento, a pesar de su lógica no esperado por mí, acabó, no precisamente de una manera agradable, mis vacilaciones. Una mañana se presentaron en mi hotel dos caballeros, de parte del marqués de Vernay. Venían a provocarme a un duelo a pistola en condiciones graves. Yo acepté, desde luego; tenía la seguridad de que no me había de pasar nada. (CORDE. P. Baroja, Las inquietudes de Shanti Andía, 1911)

Este carácter permite su aparición en el ámbito dialógico, como en (7), donde no es una locución marcadora, sino una fórmula lógica epistémica de carácter evidencial que permite mostrar afirmación o acuerdo ${ }^{18}$ :

(7)

En el Círculo Democrático los demócratas, ascendidos a prohombres, lo miran, insistentemente, por miedo a que se les pierda.

— ¿Qu'es eso, cómo andaremos por allí?

-Muy bien; espero que saquemos las mayorías.

- ¿Por los dos colegios?

- Sí, sí, desde luego.

(CORDE. Juan Antonio Zunzunegui, El Chiplichandle. Acción picaresca, 1940)

17 Así lo han manifestado, entre otros, C. Fuentes Rodríguez, «Desde luego, por supuesto, naturalmente», Sociolingüística andaluza, Estudios sobre el enunciado oral, 8, 1993, págs. 99126; y Pérez Canales, «Marcadores de modalidad epistémica: en efecto, efectivamente, desde luego, por supuesto...», en Casado Velarde, M. et alii, eds., Análisis del discurso: lengua, cultura, valores, Madrid, Arco/Libros, vol. II, 2006, págs. 1525-1540.

18 Sobre estos valores, véase B. Alvarado Ortega, Las fórmulas rutinarias del español: teoría y aplicaciones, Frankfurt, Peter Lang, 2010. 
Así pues, desde luego ha gramaticalizado tanto su forma (fijación) como su significado (idiomaticidad). En cuanto a la fijación, se ha reanalizado como marcador del discurso, y desde la posición habitualmente posverbal de los complementos circunstanciales, ha adquirido una posición relativamente libre dentro del enunciado. En cuanto a la idiomaticidad, los valores temporales se han debilitado en favor del establecimiento de consecuencias discursivas entre los enunciados presentes (reformulación); y desde aquí, se ha especializado en el reforzamiento del enunciado en el que se inserta, que se muestra como evidente (operador). Este significado facilita la adquisición de nociones de intersubjetivación, como la intensificación de la propia opinión (nivel monológico) o del acuerdo (nivel dialógico) (fórmula):

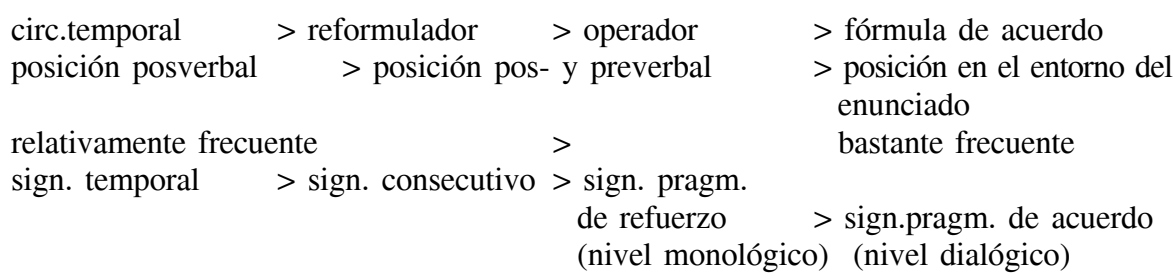

FIGURA 2. La gramaticalización de desde luego

Todo ello conlleva que el complemento circunstancial se haya convertido, en primer lugar, en una locución marcadora con funciones de reformulación o de refuerzo $\mathrm{y}$, en segundo lugar, en una fórmula rutinaria para mostrar acuerdo.

\section{LA SUBJETIVACIÓN Y LAS FÓRMULAS RUTINARIAS}

Hemos presentado un primer análisis de una locución a partir de su gramaticalización como marcador del discurso. Ahora bien, la gramaticalización desde el punto de vista funcional no se entiende sin la subjetivación. Como decíamos más arriba, este concepto ha sido desarrollado por E.C. Traugott a lo largo de diversos trabajos. Se define como:

un fenómeno gradual, por el cual formas y construcciones que inicialmente expresaban, en primera instancia, significados concretos, léxicos y objetivos, llegan a realizar, a través de un uso repetido en contextos sintácticos locales, funciones progresivamente más abstractas, pragmáticas y basadas en el emisor ${ }^{19}$.

19 E. C. Traugott, «Subjectification in grammaticalization», en D. Wright y D. Stein, eds., Subjectivity and Subjectivisation, Cambridge, CUP, 1995, págs. 31-54. (Traducción de M. ${ }^{\mathrm{a}}$ J. Cuenca y J. Hilferty, Introducción a la lingüística cognitiva, Barcelona, Ariel, 1999, pág. 163). 
De acuerdo con ello, la hipótesis del cambio supone que del significado léxico se pasa al significado pragmático; del significado concreto, al significado abstracto; y de expresar nociones concretas, a interiorizar un significado subjetivo. En el caso que ahora nos ocupa, dicho significado es el irónico que una fórmula como estaría bueno ha interiorizado. Así, con el fin de ilustrar dicha evolución, distinguimos tres estadios para estaría bueno ${ }^{20}$ que van de los significados más léxicos, concretos y objetivos a los más pragmáticos, abstractos y basados en el emisor. En concreto, la construcción ha evolucionado desde un valor temporal hacia un valor de probabilidad que se infiere por una implicatura escalar. Este proceso ha permitido crear un nuevo significado codificado, basado en la ironía ${ }^{21}$.

Fase I: Estaría bueno presenta un significado léxico, concreto y objetivo y la construcción tiene un claro valor temporal. Se refiere a situaciones de salud en las que, gracias a algún remedio, el paciente se pondrá bien en un momento cercano del futuro. La aparición del condicional viene motivada a menudo por la consecutio temporum necesaria en el discurso indirecto. La falta de fijación conlleva que el adjetivo bueno cambie de género y de número. Ya que se valora que el paciente se curará en un momento del futuro cercano, no es extraño que la combinación aparezca con adverbios como luego, después o presto, como se ilustra en (8):

(8)

Luego me partí en demanda del dotor Alderete, para que lo visitara, y lo hallé que andava ya visitando, y me dixo que no tenía neçesidad de inovar cosa alguna, mas de que tomase aquellas píldoras tres días ar[r]eo, y al cabo dellos él lo vería y proveería lo neçessario. Y, como vide aquello, dexélo y fuime a buscar a Cartagena, que tanbién lo hallé visitando. Y, entendido por él la neçesidad y afeto con que lo llamava, luego dexó el hilo y orden que de sus enfermos llevava y se bolbió conmigo y lo vido y visitó con mucha solenidad, dándole buenas esperanças de su salud, porque yo ansí se lo avía pedido. Y le dixo que, si podía estar tan solos ocho días sin beber agua, que luego estaría bueno, y para ello le dexó veinte remedios de lavatorios y píldoras que truxese en la boca para matar la sed, que venido al fallo más se la acresentavan. Y, al cabo, le pareçió la enfermedad mortal y que no tenía remedio. (CORDE, J. Méndez Nieto, Discursos medicinales, 1606-1611)

20 Tal combinación se relaciona con otra similar, estamos buenos, de manera que aparece en el corpus una mezcla de las dos, estaríamos buenos. Ahora bien, tales combinaciones muestran un comportamiento diferente a estaría bueno, la forma más fija, y se relacionan con las fórmulas sinonímicas estamos apañados y estamos arreglados. Estas últimas evidencian en el corpus un grado menor de gramaticalización.

21 Para más datos acerca del tratamiento que recibe aquí la ironía puede consultarse L. Ruiz Gurillo y X. A. Padilla García, eds., Dime cómo ironizas y te diré quién eres: una aproximación pragmática a la ironía, Frankfurt, Peter Lang, 2008. 
Cuando la sintaxis no exige la forma condicional, el verbo se encuentra en futuro. Así lo vemos en (9) dentro de un periodo de discurso directo:

(9)

Y yo confieso que muchas veces me ha sucedido que, como he tenido el corazón tan despegado de todo, el que en otro tiempo lloraba las enfermedades de los frailes con estremo, ahora con mucho despego, viendo que yo no podía más y que esta obra no era mía, viendo los enfermos les decía: hermano, rece y luego estará bueno, prométale a Dios ser muy sancto; (CORDE, San Juan Bautista de la Concepción (Juan García Gómez), La regla de la orden de la Santísima Trinidad, 1606).

Por otro lado, bueno no solo se aplica a la salud, sino también a otras circunstancias como el sabor de los platos o la madurez de los alimentos. Así se encuentra en el ejemplo (10), donde observamos además que el enunciado se inscribe en una situación hipotética:

\section{(10)}

Estas carpas hechas pedaços, y fritas, y assentadas sobre vna sopilla de pan tostado (...) y quando la manteca esté derretida, y la salsa esté blanca, échala por encima de las carpas, de manera que ande bien bañada la sopa. Esta se llama salsa de brugete: y si coziesses vnos espárragos, y luego freírlos, y assentarlos entre las carpas, y la sopa, suele salir muy buen plato. Si echasses dulce a la sopa de las carpas estarán buenas. (CORDE, F. Martínez Motiño, De cozina, pastelería, vizcochería y conseruería, 1611)

Fase II: Estaría bueno evoluciona hacia un valor de probabilidad y pasa de señalar distancia temporal a marcar distancia epistémica ${ }^{22}$. Con estaría el hablante se distancia con respecto a la proposición que enuncia, ya que frente al miembro fuerte de la escala, está, se manifiesta cierta probabilidad ante los hechos. La consecución o no de los hechos lleva implícita la negación de lo expresado. Dicho valor epistémico se considera una implicatura conversacional que se debe al Principio de Cantidad de Horn ${ }^{23}$. Además, bueno es un término valorativo que si sitúa en una escala polar (bueno-malo).

Esta combinación, de carácter libre, presenta sintácticamente un sujeto. Este sujeto, simple o proposición sustantiva introducida por que, recoge información aparecida con anterioridad, ya de manera explícita, ya como anáfora, o alude a una información que se ha de inferir del contexto.

Así se observa en (11), donde el sujeto, de carácter fórico (todo eso), recoge la información del contexto lingüístico inmediato. Asimismo, vemos cómo

\footnotetext{
22 Esta evolución se fundamenta en la cadena de gramaticalización que explica los cambios desde valores temporales a modales: persona $>$ objeto $>$ proceso $>$ espacio $>$ tiempo $>$ cualidad $(\mathrm{B}$. Heine, U. Claudi y F. Hünnemeyer, «From cognition to grammar: Evidence from African languages», en E. C. Traugott y B. Heine, eds., Approaches to gramaticalización, op. cit., págs. 149-188.

23 L. H. Horn, A Natural History of Negation, Chicago, CSLI Publications, 2001.
} 
se presentan contextualmente ambos extremos de la escala al contraponer lo que sería bueno o adecuado si se cumpliese la prótasis, a lo que es malo y ocurrirá realmente:

(11)

Saquemos otras poquitas de consecuencias de este puntito de doctrina.

Y sea la primera, que ni VV. ni todos sus maestros los filósofos, ni todos aquellos, de quienes lo han aprendido, estan á los legítimos principios, cuando para reformar, como VV. le llaman, ó para abolir, como los rancios decimos, la verdadera religion, alegan por una parte la ignorancia y desórdenes del clero, y por otra las luces, la sabiduría, la política, y si VV. quieren, hasta los milagros de los publicistas y filósofos. Todo eso estaria bueno, si el mérito ó demérito de los hombres hubiese de contribuir á la obra; pero todo está muy malo, porque la obra, no depende de ellos. Dios es el que corre con ella (...). (CORDE, Fray Francisco Alvarado, Cartas críticas del filósofo Rancio, 1811)

Por tanto, se infiere gracias a la implicatura escalar una situación probable (todo esto estaria bueno, si el mérito o demérito de los hombres hubiera de contribuir a la obra), que se cancela en el contexto (pero todo está muy malo, porque la obra, no depende de ellos). En (11) no resulta difícil inferir lo que el escritor quiere transmitir: que no sería bueno que los comisionados reformen atendiendo a los asuntos humanos y no tengan en cuenta los asuntos de Dios.

Este valor de distancia epistémica, asociado en concreto a estaría, se va generalizando, gracias a su aparición en construcciones donde el hablante/escritor no puede comprometerse con la verdad del enunciado. Por otra parte, no es extraño que la combinación venga precedida por marcadores como pues o anda que. En (12) aparece combinado con pues y presenta una proposición sustantiva que contiene lo que se considera adecuado que haga un gobernador, velar por la moral pública. Ahora bien, la combinación pues estaría bueno que ya aparece cargada del sentido negativo, propio de la estructura irónica. Junto a la fórmula encontramos otros indicadores irónicos como la anteposición de un término valorativo en la exclamación (;Bonita andaría la sociedad!) o la estructura sintáctica con ni (Ni que estuviéramos entre salvajes). Nótese, además, que la construcción se inscribe en un periodo hipotético:

(12)

Y no dejaron de molestarle también y entorpecerle ciertas disensiones domésticas, pues Refugio, que ya se estaba dando pisto de gobernadora, y se había despedido de sus amigas con ofrecimientos de protección a todo el género humano, se quedó helada cuando su señor le dijo que no la podía llevar... Pucheros, lloros, apóstrofes, quejas, gritos...

-Pero, hija de mi alma, hazte cargo de las cosas; no seas así. ¿No comprendes que no me puedo presentar en mi capital de provincia con una mujer que no es mi mujer? ¡Qué diría la alta sociedad, y la pequeña sociedad también, y 
la burguesía!... Me desprestigiaría, chica, y no podríamos seguir allí. Esto no puede ser. Pues estaría bueno que un gobernador, cuya misión es velar por la moral pública, diera tal ejemplo. ¡El encargado de hacer respetar todas las leyes, faltando a las más elementales!... ¡Bonita andaría la sociedad, si el representante del Estado predicara prácticamente el concubinato! Ni que estuviéramos entre salvajes... Convéncete de que no puede ser. Tú te quedas aquí y yo te mandaré lo que vayas necesitando... Pero lo que es allá no me pongas los pies... porque si lo hicieras, tu chachito se vería en el caso de cogerte... ya sabes que tengo mucho carácter... de cogerte y mandarte para acá por tránsitos de la Guardia civil. (CORDE, B. Pérez Galdós, Fortunata y Jacinta, 1885-1887)

Fase III: Estaría bueno presenta un significado pragmático, abstracto y basado en el emisor. Se comporta como un término de polaridad negativa. Por un lado, la aparición repetida en contextos irónicos ha influido en la codificación de las inferencias negativas que se derivaban conversacionalmente de estaría; por otro, se han gramaticalizado las inferencias derivadas de la escala polar a la que pertenece bueno. Se aplica aquí el principio de negación de los extremos ${ }^{24}$, según el cual se obtiene por implicatura el significado correspondiente al otro extremo de la escala polar. De acuerdo con tal principio, se invierte la polaridad de la frase y, en consecuencia, el significado es el de "no es bueno' 25 .

La fórmula suele encontrarse como coda a otra información y con frecuencia aparece combinada con pues. Prescinde en muchos casos de la proposición subordinada que era su sujeto. Los enunciados que antes expresaban de manera explicíta lo que sería bueno, ahora se sobreentienden con la aparición de la fórmula. Ya que se trata de una UF que ha fijado su forma y su significado irónico, actúa como un indicador de que el enunciado en el que aparece puede ser irónico.

En (13) encontramos la fórmula como elemento parentético entre comas y no presenta la proposición con que. Se inscribe además en un enunciado propiamente irónico, donde el hablante ironiza sobre el desconocimiento que tiene su oyente, una mujer, de los nuevos aparatos de aire caliente y frío venidos del extranjero:

(13)

Esto es más fácil de lo que parece, sí, señorita, no hay más que apretar aquí, ¿ve? y ya está. Zumbando. ¿No lo nota? Burrr... Es aire de la calle, para ventilar, para renovar, bueno, como esto es una oficina muy bien, puede usted decir conmutar, sustituir, trasformar, en fin, como usted quiera. El caso es

24 I. Bosque, Sobre la negación, Madrid, Cátedra, 1980.

${ }^{25}$ Como indica S. Rodríguez Rosique, «Una propuesta neogriceana», en L. Ruiz Gurillo y X. A. Padilla García, eds., Dime cómo ironizas y te diré quién eres, op. cit., el refuerzo de la negación se puede interpretar en sentido irónico cuando los términos constituyen extremos de una escala. Es lo que ocurre con estaría bueno. 
que hay que apretar aquí. No, hombre, no ahí no, ése es para dar calor. ¿Cómo que si este aparato da calor? Este aparato da de todo, estaría bueno, pero, oiga, usted ¿dónde vive que no conoce estos aparatos? Estos aparatos son lo que hay que ver, caramba, si lo sabré yo. (CORDE, A. Zamora Vicente, A traque barraque, 1972)

Este uso no dista temporalmente de los que se documentan en el corpus sincrónico CREA. Cada vez resulta más frecuente su empleo como coda con carácter de operador pragmático. De este modo, afectaría a las capacidades argumentativas del enunciado en el que se inserta. En palabras de E. C. Traugott y R. B. Dasher ${ }^{26}$, funcionaría en ocasiones como un matizador que actúa en la relación entre hablante y oyente, esto es, como un elemento de intersubjetivación. Así pues, el camino hacia la gramaticalización nos conduce de una fórmula rutinaria a un elemento de intersubjetivación. Su significado es el de mostrar oposición a algo, como nos indica el DRAE ${ }^{27}$. Esa idea de oposición es, en realidad, el significado irónico que se ha codificado. Así, el significado que esperaríamos es el de 'sería bueno'. Pero no es este el significado que se extrae de esa fórmula, sino que «se usa para comentar irónicamente la mala situación en que se encuentra o se encontraría alguien o algo, o lo equivocado de una previsión o de una apreciación» ${ }^{28}$. Así lo vemos en un contexto como el de (14). Asistimos a una pelea en la que un muchacho sostiene en vilo a un enano y pide consejo sobre lo que ha de hacer con él. Obsérvese cómo se inscribe en un entorno negativo:

(14)

El enano dio un salto superfluo de enano, con la intención quizá de alcanzar al muchacho con el rebenque lo más arriba posible. Pero el muchacho, que había dejado el pozal en el suelo, esquivó aquella grotesca acometida y casi sostuvo al enano en vilo por debajo del brazo. El enano acezaba igual que un podenco y logró zafarse a la vez que azotaba de refilón las corvas del muchacho. Y en eso acudieron dos de los hombres que habían entrado en el despacho de vinos y apartaron, entre defectuosos consejos, a los desiguales contendientes.

—Un maricón — dijo el enano, cuya palidez había adquirido la tonalidad del mandil-. Eso es lo que tú eres, un maricón.

— ¿Qué hago con él? —le preguntó a nadie el muchacho-. No hace más que buscarme y yo como si no fuera conmigo. A ver qué hago con esta cucaracha.

26 Op. cit.

27 RAE, Diccionario de la lengua española, Madrid, Espasa, edición en CD-Rom, versión $1.0,2003$.

${ }^{28}$ M. Seco et alii, Diccionario fraseológico documentado del español actual, Madrid, Aguilar, 2004, pág. 209. 
-Déjalo - dijo uno de los hombres-. No te vas a liar con él, estaría bueno. El enano permanecía delante del mostrador, haciendo oscilar el rebenque en el aire. No decía nada, pero tenía un filo de navaja atravesándole los ojos, que, bizqueaban con una ficticia ferocidad de ocelos. Y ya no hubo más conatos de reyerta aquella mañana. (CREA, J. M. Caballero Bonald, Toda la noche oyeron pasar pájaros, 1981)

Aquí significa "no es adecuado que te líes con el enano que te acusa de maricón'. Es decir, en este contexto habría codificado plenamente las inferencias negativas.

$\mathrm{Su}$ comportamiento, frente a lo que pudiera pensarse, no es únicamente contextual, sino que la codificación de tal significado negativo (irónico) tiene repercusiones sintácticas. Como se observa en (14'), se comporta como un término de polaridad negativa de carácter débil:

No te vas a liar con él, estaría bueno

Estaría bueno que te liaras con él

En el primer caso, se sitúa en la posición de coda, la más frecuente en los ejemplos analizados en el CREA. En la segunda, su aparición en primera posición anula el activador negativo.

Por lo tanto, estaría bueno muestra una evolución desde significados léxicos, concretos y objetivos a otros más pragmáticos, abstractos y basados en el emisor, que se resumen del siguiente modo:

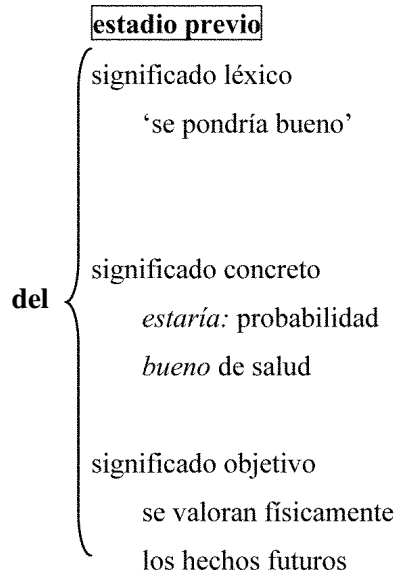

\author{
estadio actual \\ significado pragmático \\ negación de la proposición: \\ 'no es bueno' \\ significado abstracto \\ término de polaridad negativa \\ de carácter débil \\ significado basado en el emisor \\ actúa como una fórmula rutinaria \\ que facilita un entorno irónico
}

FIGURA 3. La subjetivación en estaría bueno 


\section{LA TEORÍa DEL CAMBIO SEMÁNTICO A PARTIR DE INFERENCIAS ASOCIADAS Y LAS LOCUCIONES VERBALES}

La propuesta de la subjetivación antes vista ha sido convenientemente integrada en los últimos años en la Teoría del Cambio Semántico a partir de Inferencias Asociadas (Invited Inferencing Theory of Semantic Change, IITSC). El objetivo principal de esta propuesta es dar cuenta de la convencionalización de los significados pragmáticos y de su consiguiente reanálisis como significados semánticos ${ }^{29}$. Para explicar tal relación entre estos niveles lingüísticos, el modelo bebe en la pragmática de Grice, y más concretamente en la pragmática neogriceana desarrollada por L. Horn o S. C. Levinson ${ }^{30}$.

De este modo, y como se resume en la figura 4, se propone una evolución desde los significados codificados a los que se asocian determinados significados que se infieren como implicaturas conversacionales particularizadas (ICP) (Estadio I) que, con el paso del tiempo y con su aparición en determinados contextos, se van convirtiendo en implicaturas conversacionales generalizadas (ICG). Así pues, los significados pragmáticamente polisémicos van a ir desarrollando, gracias a las implicaturas, significados semánticamente polisémicos (codificados) (Estadio II):

Significado de enunciado tipo

Convencionalización de las ICP en ICG

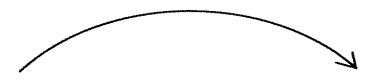

El hablante-escritor/oyente-destinatario restringe el peso de la ICP

(usos preestablecidos, prominencia, relevancia, subjetividad, etc.)

\section{フ}

Significado de enunciado

El hablante-escritor explota las ICP

de manera innovadora en la cadena de habla
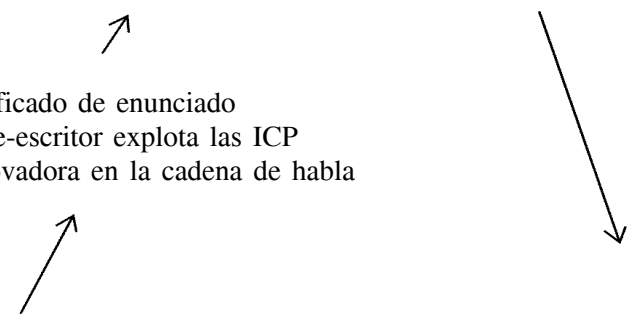

Estadio I. Significado codificado

$>\quad$ Estadio II. Nuevo significado codificado (semantización)

FIgURA 4. Modelo de la Teoría del Cambio Semántico a partir de Inferencias Asociadas (IITSC) (E. C. Traugott y R. B. Dasher, op. cit., pág. 38, de acuerdo con E. C. Traugott), traducido al español -la traducción es nuestra-.

${ }^{29}$ E. C. Traugott y R. B. Dasher, op. cit., pág. 35.

${ }^{30}$ L. H. Horn, op. cit., S. C. Levinson, Presumptive meanings, Cambridge, MIT Press, 2000. 
Tales cambios, que son unidireccionales ${ }^{31}$, se relacionan a su vez con diversos patrones de gramaticalización que Traugott y Dasher concretan en los siguientes:

condiciones de verdad

contenido

proposición

de alcance interno

no subjetivo

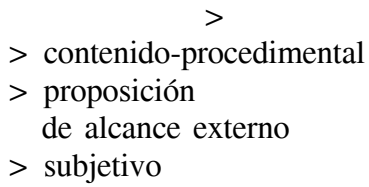

no condiciones de verdad

$>$ procedimental

$>$ elemento

de alcance discursivo

$>$ intersubjetivo

FIGURA 5. Cadenas correlativas de la direccionalidad en el cambio semántico, de acuerdo con E. C. Traugott y R. B. Dasher, op. cit., pág. 40)

Es en este contexto donde adquiere sentido la subjetivación que se asocia de manera metonímica al acto de comunicación del hablante o escritor y, más concretamente, a su actitud. Así, indican los autores que la subjetivación es el tipo principal de cambio semántico. Como veíamos más arriba, se completa con la intersubjetivación, que se subordina a ella y que no puede darse sin la primera. Supone un paso más y permite explicar determinados procedimientos de codificación de la actitud del hablante.

También se encuentra un significado codificado de carácter irónico en la locución verbal cubrirse de gloria. Hemos visto que en la fórmula rutinaria estaría bueno la codificación de la implicatura ha ocasionado la selección de las inferencias negativas de la escala. Ello ha suscitado que la única lectura de la UF irónica sea lo contrario de lo que dicen las palabras. Ahora bien, la locución verbal cubrirse de gloria no funciona a partir del principio de cantidad, sino a partir de los principios de informatividad y manera de Levinson. De este modo, la inversión de tales principios al que conduce la ironía supone que la expresión no se interprete como lo contrario de lo que se dice, sino como algo distinto a lo que se dice. El examen de los ejemplos que arrojan el CORDE y el CREA nos permite proponer una evolución gradual del significado composicional al idiomático, y de la forma más libre a la más fija; al tiempo, se observa que la combinación libre, que pertenece al lenguaje específico de la guerra, se va generalizando a todos los ámbitos. Como hemos hecho con desde luego y con estaría bueno, observaremos dichos cambios por medio de tres fases.

Fase I: el sintagma cubrirse de gloria presenta un significado literal. Se emplea con frecuencia en el lenguaje de la guerra y alude a los que salen vic-

${ }^{31}$ Numerosas han sido las críticas a la unidireccionalidad, venidas de diversas propuestas teóricas; algunas de ellas han sido asumidas por el modelo (véase por ejemplo E. C. Traugott, «Legitimate counterexamples to unidirectionality», conferencia presentada en la Universidad de Friburgo, 17 de octubre de 2001 (http://www.stanford.edu/ traugott/papers/Freiburg.Unidirect.pdf). 
toriosos en la batalla, gracias a su fuerza y a su valentía. La existencia de vencedores supone asimismo la existencia de perdedores, significado que se obtiene por implicatura conversacional particularizada.

El ejemplo (15) es el primer caso documentado que encontramos (1650). Se trata de un fragmento en el que Simón consagra a siete discípulos como obispos y los envía a extender la palabra de Dios por el mundo. En él les advierte de que velará por ellos como un guerrero y luchará contra el vicio hasta la muerte. En este contexto, el manto ha de cubrir de gloria a los discípulos antes de que Simón cese en sus trabajos en defensa de la virtud:

(15)

Onrrarán las ciudades escogidas

de sus Patronos las felizes muertes,

las sitios, los annales, las istorias,

los huessos, las zenizas, las memorias.

Primero subiréys al trono santo

que yo dé fin a la mortal contienda,

y antes os cubrirá de gloria el manto

que cessen los trabajos de mi senda.

(CORDE, Pedro de Solís y Valenzuela, El desierto prodigioso y prodigio del desierto, 1650).

La expresión que nos ocupa se manifiesta como un sintagma libre desde esta época hasta la actualidad en los géneros textuales relacionados con la guerra (historiografía, épica bélica, etc.). De este modo, el circunstancial puede coordinarse a otros circunstanciales:

(16)

Confunde sus almas, en un velo cubriéndolas de gloria y de ventura.

(CORDE, José de Espronceda, El diablo mundo, 1840-1941).

O admite modificadores como ocurre en (17). En este caso, es el caudillo el que se cubre de gloria, frente a los pueblos sometidos que quedan en lugar opuesto:

(17)

Observando estas y otras precauciones que se omiten porque se deducen fácilmente de las indicadas, no se harán seguramente aquellas célebres conquistas que aturden por su rapidez, y cubren de gloria tan brillante al caudillo que las manda, no se esclavizarán pueblos enteros por un rasgo solo de valor y de osadía; (CORDE, Juan Romero Alpuente, Historia de la Revolución de España, 1831).

En otros contextos, convive con verbos como morir, huir o con combinaciones como sufrir la derrota. También se encuentra en estructuras contrapuestas (como la disyuntiva o la adversativa). Así lo encontramos en (18), donde 
cubrirse de gloria aparece contrapuesto a morir, por lo que se infiere del contexto que estas son las dos opciones que tiene el que lucha en el campo de batalla frente a los franceses:

\section{(18)}

En el primer combate usted se cubrirá de gloria o morirá, y de una u otra manera quedará bien puesto a los ojos de su rival y los de esa señorita (...). (CORDE, I. M. Altamirano, Clemencia, 1869).

Fase II: Cubrirse de gloria no se emplea de manera recta, sino como una alusión del hablante, de carácter metalingüístico, hacia la situación bélica en la que existe un vencedor, pero también un perdedor. Se encuentra en contextos donde se alude a la guerra, pero que pertenecen a géneros textuales más amplios $^{32}$.

Así se observa en (20). Doña Flora está hablando de los desastres de la guerra y del honor nacional. En este contexto menciona una gran derrota, la del cabo de Finisterre, pero no quiere volver a oír la solución que, para el amo, hubiera servido para alcanzar la gloria. En este contexto en el que se recuerda una España victoriosa, pero también una España derrotada o traicionada por sus aliados, se aplica cubrirse de gloria a una persona que no vale para el combate. El empleo en este contexto de la locución cubrirse de gloria tiene una clara intención irónica que doña Flora dirige hacia su interlocutor, el amo, pues ya ha contado repetidas veces la misma historia de honor y sangre:

- Verdad es que el honor nacional es lo primero, y es preciso seguir adelante para vengar los agravios recibidos. No me quiero acordar de lo del cabo de Finisterre, donde, por la cobardía de nuestros aliados perdimos el Firme y el Rafael, dos navíos como dos soles; ni de la voladura del Real Carlos, que fue una traición tal, que ni entre moros berberiscos pasaría igual; ni del robo de las cuatro fragatas, ni del combate del cabo de...

—Lo que es eso... — dijo mi amo interrumpiéndola vivamente-. Es preciso que cada cual quede en su lugar. Si el almirante Córdova hubiera mandado virar por...

—Sí, sí, ya sé — dijo doña Flora, que había oído muchas veces lo mismo en boca de mi amo- - Habrá que darles la gran paliza, y se la daréis. Me parece que vas a cubrirte de gloria. Así haremos rabiar a Paca.

- Yo no sirvo para el combate - dijo mi amo con tristeza. Vengo tan sólo a presenciarlo, por pura afición y por el entusiasmo que me inspiran nuestras queridas banderas. (CORDE, B. Pérez Galdós, Trafalgar, 1873).

De igual modo, en (20) la marquesa ironiza sobre el general Bernal, que ella misma considera un «general improvisado». Obsérvese cómo Valentín em-

\footnotetext{
32 Conviene no olvidar que estos nuevos usos conviven durante toda la historia de la locución con los empleos rectos que se restringen al lenguaje de la guerra.
} 
plea la expresión en sentido recto y a continuación la marquesa la usa como indicador de la ironía, junto a otros indicadores como la repetición de gloria o la estructura sintáctica exclamativa ;buena gloria te dé Dios! Con todo ello, muestra su desacuerdo ante la idea de que el general Bernal le conviene a su sobrina como marido:

(20)

Marquesa. Basta, basta de pierna, y óigame usted.- Mi sobrina la duquesa del Puerto ha dado en la flaqueza de honrar con su aprecio á un hombre que, á decir lo que siento, no lo merece.

Valentín. ¡Señora!

Marquesa. ¡No me interrumpa!- Usted sin duda ignora que uno de los ascendientes del duque del Puerto murió en la toma de Sevilla, al lado del santo rey D. Fernando.

Valentín. Si moriría: eso debe usted saberlo mejor que yo. Yo no estuve en esa batalla.

Marquesa. ¡Es que yo tampoco!

Valentín. No, no digo yo que usted estuviera.

Marquesa. ¡Me gusta la especie!

Valentín. Yo donde he estado es en otros combates, donde el general Bernal se cubrió de gloria.

Marquesa. ¡Buena gloria te dé Dios! ¡Ahora todo el mundo se cubre de gloria! Valentín. Lo que es él, me parece que no habrá quien dude en España... Marquesa. Bien: no disputo su gloria.

Valentín. Y hace usted bien.

Marquesa. Pero me confesará usted que no por eso está á menos distancia de mi sobrina.

Valentín. ¡No, señora; no la confesaré á usted tal!

Marquesa. ¡Oiga y no me interrumpa tanto!

Valentín. ¡Y usted también hágame el favor de no ofender al general Bernal! Marquesa. No faltaba más sino que la marquesa de Estepona tuviese que guardar respeto á un general improvisado. (CORDE, Ventura de la Vega, $A$ muerte o a vida o La escuela de las coquetas. Comedia en tres actos, arreglada al español, 1842).

Tanto en (19) como en (20), cubrirse de gloria se convierte en una expresión marcada que, indica, por tanto, una situación marcada (principio de manera). La polisemia que rodea a la expresión afecta también al principio de informatividad. Su empleo en clave irónica conlleva la inversión de manera particularizada de las inferencias que se derivan de dichos principios y permite comprender en (19) que el amo va a actuar como no debe y en (20) que la marquesa no guarda respeto al general Bernal por su condición de general.

Fase III: El significado irónico se convierte en un nuevo significado codificado gracias a la fraseologización del sintagma. De este modo, aunque previsiblemente el sintagma libre sigue funcionando en el lenguaje de la guerra, la 
locución ha fijado su forma y presenta un significado idiomático de carácter irónico que se aplica a diversas situaciones, no solo a las que tienen que ver con la guerra.

En (21) cubrirse de gloria se aplica al fiscal general del Estado que no está actuando como sería esperable de acuerdo con el cargo que ocupa:

(21)

Se moviliza al fiscal general del Estado para que impida la investigación del juez instructor del caso Marino Barbero. ¿Te acuerdas de Eligio Hernández, el fiscal particular del Gobierno, aunque nombrado, y mal nombrado fiscal general del Estado? Se cubrió de gloria en este y en otros muchos casos de similares circunstancias. (CREA, C. Almeida, Carta abierta a una política honrada sobre la corrupción, 1995).

En resumen, la locución verbal cubrirse de gloria ha evolucionado de sintagma libre a sintagma fijo y ha cambiado su significado composicional de 'cubrirse de gloria' por el significado idiomático de carácter irónico de 'meter la pata'. La evolución supone, por un lado, que los significados contextuales se han ido haciendo progresivamente más generales; en este caso, que las acciones que se realizan para obtener honor y gloria conllevan también la desgracia o la derrota de otros. Por otro lado, del empleo restringido en el lenguaje de la guerra se ha pasado a un uso generalizado. Retomamos la propuesta de las IITSC de E. C. Traugott y R. B. Dasher para explicar las evoluciones que afectan a cubrirse gloria:

\section{Significado de enunciado tipo}

Cubrirse de gloria se emplea de manera marcada en situaciones marcadas irónicas como indicador (principio de manera)

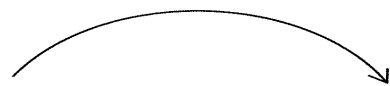

El hablante-escritor/oyente-destinatario restringe el peso de la ICP

El hablante-escritor emplea de forma marcada o prominente cubrirse degloria; alude de manera metalingüística a una situación de guerra sobre la que ironiza (principio de informatividad)

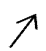

Significado de enunciado

'De la existencia de vencedores se infiere la existencia de vencidos'

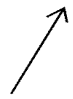

Estadio I. Significado codificado 'cubrirse de alabanza ante un hecho heroico'

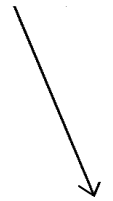

$>$ Estadio II. Nuevo significado codificado (semantización) 'meter la pata'

FIgURA 6. Aplicación de la Teoría del Cambio Semántico a partir de Inferencias Asociadas (IITSC) a la evolución de la locución irónica cubrirse de gloria 


\section{CONCLUSIONES}

En este trabajo hemos mostrado cómo resulta posible estudiar diversas unidades fraseológicas a partir de los principios de gramaticalización. Las locuciones marcadoras, representadas por desde luego, se han analizado tomando como base la cadena de gramaticalización propuesta por E. C. Traugott para los marcadores del discurso. Hemos visto cómo la estructura ha evolucionado desde los valores temporales hasta actuar como reformulador y como operador e, incluso como elemento de intersubjetivación cuando funciona como fórmula de acuerdo. En cuanto a las fórmulas rutinarias, estaría bueno ha sido analizada de teniendo en cuenta el proceso de subjetivación e intersubjetivación sufrido. Manifiesta una evolución desde significados léxicos, concretos y objetivos a otros más pragmáticos, abstractos y basados en el emisor, entre los que destaca su función como término de polaridad negativa y su función como indicador irónico. Por último la Teoría del Cambio Semántico a partir de Inferencias Asociadas ha supuesto la base para mostrar la evolución de la locución verbal cubrirse de gloria hacia significados irónicos. En conclusión, el enfoque adoptado en este trabajo permite sostener que los principios de gramaticalización otorgan un nuevo carácter a los principios fraseológicos de fijación e idiomaticidad, pues los integran en una propuesta general del cambio lingüístico. 